Revue d'histoire de l'Amérique française

REVUE D.HISTOIRE DE L'AMÉRIQUE FRANÇAISE

\title{
Enseignement de la philosophie au Petit Séminaire de Québec (1765-1880)
}

Marc Lebel

Volume 18, numéro 3, décembre 1964

URI : https://id.erudit.org/iderudit/302391ar

DOI : https://doi.org/10.7202/302391ar

Aller au sommaire du numéro

Éditeur(s)

Institut d'histoire de l'Amérique française

ISSN

0035-2357 (imprimé)

1492-1383 (numérique)

Découvrir la revue

Citer cet article

Lebel, M. (1964). Enseignement de la philosophie au Petit Séminaire de Québec (1765-1880). Revue d'histoire de l'Amérique française, 18(3), 405-424.

https://doi.org/10.7202/302391ar d'utilisation que vous pouvez consulter en ligne.

https://apropos.erudit.org/fr/usagers/politique-dutilisation/ 


\section{ENSEIGNEMENT DE LA PHILOSOPHIE AU PETIT SÉMINAIRE DE QUÉBEC * \\ $(1765-1880)$}

\section{INTRODUCTION}

L'histoire de l'enseignement secondaire est l'une des provinces les plus intéressantes de l'histoire des mentalités. Elle ouvre de larges avenues sur les années capitales de l'adolescence; au collège, l'adolescent acquiert connaissances et habitudes, rencontre maîtres et camarades; il grandit, il devient proprement ce qu'il est.

En étudiant l'histoire de l'enseignement de la philosophie au Petit Séminaire de Québec, nous n'avons pas la prétention de revêtir la chlamyde du philosophe. Nous nous sommes attaché aux faits d'enseignement. Nous avons cherché à comprendre ce qu'enseignent les professeurs, à connaître les exercices auxquels ils soumettent leurs élèves, à découvrir les lectures des uns et des autres.

Nous avons fait la plus grande partie de nos recherches aux archives du Séminaire de Québec. Nous avons examiné tour à tour les plans d'éducation et les programmes d'études, les catalogues de bibliothèque et les annuaires; nous avons dépouillé L'Abeille et les procès-verbaux de deux académies; nous nous sommes longuement attardé sur des cahiers de notes laissés par des écoliers et des professeurs, ainsi que sur les manuels par

* Thèse de licence ès lettres (Université Laval, Québec). L'auteur tient à exprimer sa reconnaissance à M. Claude Galarneau, directeur de l'Institut d'histoire de l'Université Laval, qui a dirigé ce travail. 
eux utilisés. Les archives du Séminaire possèdent une quarantaine de cahiers manuscrits de philosophie ayant appartenu à des écoliers et à des professeurs; ils sont bien conservés et répartis tout au long de la période que nous étudions. A la bibliothèque de l'Université Laval, nous avons retrouvé et les manuels dont s'inspirent les professeurs dans la préparation de leurs cours et les manuels mis entre les mains des écoliers, quand cesse le cours magistral. Documentation abondante, variée et, dans sa partie essentielle, à peu près entièrement rédigée en latin.

Le présent travail commence avec les débuts de l'enseignement classique au Séminaire et s'arrête au lendemain de la promulgation de l'encyclique Aeterni patris. Il comprend trois parties: le temps des compendium (1765-1800) ; l'époque de Jérôme Demers (1800-1850); la montée du thomisme (1850-1880). L'an 1800 marque à tous égards une étape: l'abbé Jérôme Demers entreprend une longue et brillante carrière de professeur; l'enseignement de la philosophie est solidement établi ; aux compendium succèdent de volumineux cahiers, puis un manuel canadien. Le demi-siècle voit la fin de l'emploi du manuel de l'abbé Demers; on remanie les programmes et l'on s'achemine insensiblement vers la restauration du thomisme prescrite par Léon XIII en 1879 .

Notre sujet n'est pas tout à fait neuf. Deux articles s'y rapportent, le premier écrit par Mgr Louis-Adolphe Paquet ${ }^{1}$, le second par l'abbé Maurice Roy. ${ }^{2}$ Ce sont deux travaux de pionniers attentifs seulement à la doctrine et soucieux de mesurer les écarts de pensée en face du thomisme. Quant au livre de Hermas Bastien ${ }^{3}$, il décrit surtout le sort de la philosophie au Canada français pendant le premier tiers du XXe siècle.

1 Coup d'oil sur l'histoire de l'enseignement de la philosophie traditionnelle au Canada, in MSRC (troisième série) XI (1917): 37-60.

2 Pour l'histoire du thomisme au Canada, in Essais et bilans, Journées thomistes I (Ottawa, 1935) : 17-28.

3 L'enseignement de la philosophie, Vol. I: Au Canada français (Montréal, 1936). 


\section{Chapitre Premier}

\section{LES DÉBUTS : LE TEMPS DES COMPENDIUM (1765-1800)}

C'est en 1765 que le Séminaire de Québec, prenant la relève du Collège des Jésuites, se met à dispenser un cours d'études classiques. Sa tâche est ardue: former des professeurs, élaborer un programme d'études, acheter des livres de classes, bref, se transformer en collège, bien que son passé ne l'y prépare guère.

A la vérité, cependant, tout n'est point à faire. Au commencement du XVIIIe siècle, le Séminaire, mécontent de l'enseignement philosophique des Pères, avait songé à organiser des leçons de philosophie pour ses pensionnaires. En 1732, il confie cette besogne à un prêtre nouvellement arrivé, Monsieur de Lamothe. Jusqu'à la Conquête, on semble avoir conservé, au Séminaire, l'habitude de faire des "répétitions" et de donner des conférences de philosophie. ${ }^{1}$ Cette sorte de tradition explique, pour une bonne part, l'intérêt porté à la philosophie dès l'ouverture des classes.

$\mathrm{Du}$ reste, la philosophie est peut-être la discipline qui jette le plus de lumières sur les débuts assez obscurs de l'enseignement classique au Séminaire. Pour comprendre la faveur relative dont jouit la philosophie, il faut tenir compte de l'influence de Rollin et de Batteux, dont on suit les préceptes, ainsi que nous l'apprend le plan d'éducation de $1790 .^{2}$

Le premier, dans son Traité de la manière d'étudier et d'enseigner les Belles-Lettres, ouvrage mieux connu sous le titre

1 Sur cette question: ASQ Lettre carton $\mathrm{M}, \mathrm{N}^{\circ} 31$; Sém. $5, \mathrm{~N}^{\circ} 50 \mathrm{a}$; Amédée Gosselin, l'Instruction sous le régime français 1635-1760 (Québec, 1911), 259 s., $274,410$.

2 Plan d'éducation du Séminaire de Québec, octobre 1790, document CXV, in RUL (novembre 1958): 272. Sur l'influence de Rollin et de Batteux au Séminaire, voir les indications données par M. Pierre Savard, Les débuts de l'enseignement de l'histoire et de la géographie au Petit Séminaire de Québec, 1765-1880, in RHAF, XV (1961): 510 s. Ajouter cependant que le Traité des Etudes apparaît en 1782 dans le catalogue de la bibliothèque du Séminaire. Sur ce catalogue, Infra, $16 \mathrm{~s}$. 
abrégé de Traité des Études, et publié de 1726 à 1728, fait l'éloge de la philosophie. Le second, auteur d'un Cours de BellesLettres ou Principes de la littérature, paru pour la première fois en 1754, reprend le Traité des Études, si bien qu'il suffit d'examiner l'ouvrage de Rollin pour savoir comment on entendait l'enseignement de la philosophie.

Professeur, principal de collège, puis recteur de l'Université de Paris, Charles Rollin a profondément marqué les milieux pédagogiques de son temps. Au dire d'un historien, il fut le "législateur incontesté de l'enseignement secondaire au XVIIIe siècle". ${ }^{3}$ Son influence s'est prolongée au-delà de la Révolution; de bons esprits, tels que Villemain et Nisard, ne lui adresseront que des éloges.

Rollin consacre un livre de son Traité des Études à la philosophie: livre assez court qu'il présente comme une "simple exhortation aux jeunes à l'étudier avec soin"." A la différence de l'histoire sur laquelle il écrit un véritable précis, Rollin se contente de montrer l'importance de la philosophie:

C'est la partie des études, dit-il, la plus importante, la plus nécessaire, la plus décisive pour les jeunes gens, et celle dont la perte se peut le moins courir et est la plus irréparable. ${ }^{5}$

Rien ne lui paraît plus funeste que l'habitude, courante alors, d'abréger le temps destiné à la philosophie ou de retirer les enfants après l'étude des belles-lettres:

Les parents qui aiment véritablement leurs enfants doivent leur faire faire le cours entier de philosophie ...

Suivant Rollin, la philosophie perfectionne la raison, règle les mœurs et orne l'esprit d'une foule de connaissances. Elle

3 Augustin Sicard, Les études classiques avant la Révolution (Paris, 1887), 41.

4 Charles Rollin, Traité des Etudes ou de la manière d'enseigner et d'étudier les Belles-Lettres (Paris, 1845), 617.

5 Ibid., 626.

${ }^{6}$ Loc. cit. 
prémunit les jeunes gens contre les faux raisonnements et surtout, elle leur inspire un profond respect pour la religion. La philosophie, d'ailleurs, conduit l'homme à Dieu :

... un des grands effets et le fruit le plus essentiel de la philosophie, c'est d'élever l'homme à la connaissance de la grandeur de Dieu ... . Le grand et important service que la bonne philosophie rend à l'homme, c'est de le disposer à recevoir avec docilité et respect tout ce que lui enseigne la révélation divine. ${ }^{7}$

Rollin observe la division traditionnelle de la philosophie en logique, métaphysique, morale et physique. Mais s'il défend l'usage de l'appareil syllogistique alors en butte à des railleries, il se montre bien disposé à l'endroit du cartésianisme à qui il attribue le "progrès extraordinaire" accompli depuis un siècle dans l'étude de la philosophie. ${ }^{8}$

Ami de Port-Royal, Rollin recommande en philosophie la lecture des Essais de Nicole et des Pensées de Pascal. Il veut qu'on mette entre les mains des écoliers l'Art de penser ou la logique de Port-Royal, ouvrage qu'il tient en très haute estime:

Je ne connais rien, dit-il, qui soit plus propre à donner aux jeunes gens de l'estime et du goût pour la philosophie, ni qui puisse leur en faire sentir tous les avantages, et même la nécessité. ${ }^{9}$

Bien plus, Rollin se félicite de voir les œuvres de Descartes et de Malebranche passer dans l'enseignement. Il loue l'habitude qu'ont prise certains collèges de faire faire par les "écoliers les plus forts" des précis de la Recherche de la vérité, des Méditations et des Principes de physique.

Rollin qui n'ignore pas l'aridité de la philosophie ${ }^{10}$, multiplie les conseils d'ordre pédagogique. Il invite les maîtres à pré-

\footnotetext{
$7 \mathrm{Ib} i d ., 640 \mathrm{~s}$.

8 Ibid., 623.

9 Ibid., 622. La bibliothèque de l'Université a conservé 5 éditions de l'Art de penser.

10 "Le passage subit de l'étude des belles-lettres à celle de la philosophie, c'est-à-dire d'un pays agréable, riant, et tout rempli de fleurs, à une région pour l'ordinaire sèche, épineuse et escarpée, rebute quelquefois les jeunes gens." Ibid., 624 .
} 
parer de bonne heure les jeunes gens à son étude; les professeurs de belles-lettres et d'éloquence, en particulier, doivent dans ce dessein habituer leurs élèves à "peser les raisons plus les paroles, à discerner partout le vrai." 11

Aux professeurs de philosophie, Rollin rappelle qu'il faut exercer les nouveaux à des matières faciles, et non point les rebuter par des syllogismes. Enfin, pour entretenir le goût des belleslettres et assurer la pure latinité des cahiers, Rollin suggère la lecture des œuvres philosophiques de Cicéron.

Rollin ne semble pas avoir été suivi sur tous les points. Jusqu'à la Révolution, la plupart des collèges et des séminaires demeurèrent fidèles à la vieille scolastique. Comme on pouvait s'y attendre, l'Encyclopédie concentra son tir sur elle. D'Alembert ne voyait dans la philosophie des collèges que le "rendezvous d'une infinité de questions inutiles". ${ }^{12}$ Il n'y eut peut-être pas au XVIIIe siècle d'enseignement plus ridiculisé que celui de la philosophie.

En dépit de la révolution scientifique qui avait aux XVIe et XVIIe siècles renversé l'autorité de l'antiquité et du moyen âge, la philosophie des collèges comportait toujours l'étude de la physique générale. Rien ne devrait jeter plus de discrédit sur elle et exciter davantage la verve des philosophes et des réformateurs que ce "tissu d'impertinences" ${ }^{13}$, que cette "étude vraiment digne du temps où l'on croyait que la nature ne pouvait agir que suivant les principes d'Aristote". ${ }^{14}$

A vrai dire, les idées cartésiennes, dont s'imprègnent les milieux ecclésiastiques depuis le début du XVIIIe siècle, se glis-

11 Loc. cit.

12 L'Encyclopédie ou dictionnaire raisonné des sciences, des arts et des métiers (Ed. 1777...), VIII: 496. Cette citation est extraite de l'article: Collège, qui contient une charge virulente contre l'enseignement de la philosophie.

13 Ibid., XXX: 287.

14 Guyton de Morveau dans son Mémoire sur l'Education publique (1764), cité par Sicard, op. cit., 199. On notera que ces querelles ont des échos canadiens: Marcel Trudel, L'influence de Voltaire au Canada (Montréal, 1945), I: 97 s., 100. 
sent peu à peu dans l'enseignement. Après avoir été combattu au XVIIe siècle, le cartésianisme, au moment où les esprits avancés s'éprennent de Locke et de Newton ${ }^{15}$, devient une doctrine officielle avec laquelle la scolastique des collèges doit s'accommoder. ${ }^{16}$

C'est dans cet arrière-plan que se poursuit l'enseignement de la philosophie au Petit Séminaire. Le plan d'éducation de 1790 nous apprend que le cours de philosophie dure deux ans et "que les classes sont alternatives:

La physique qui se faisait l'an dernier depuis le ler octobre 1789 jusqu'au 1er du présent, ne se fait point cette année, mais la logique: (c'est ainsi que nous appelons les deux années de philosophie).17

La classe de logique se consacre à la philosophie proprement dite, tandis que celle de physique s'adonne uniquement aux sciences:

$1^{\text {e }}$ année: La logique, la métaphysique et la morale.

$2^{\mathrm{e}}$ année: les Mathématiques et la Physique. ${ }^{18}$

Si l'étude des sciences est nettement distincte, dans le temps, de celle de la philosophie, il demeure qu'un seul professeur enseigne les deux disciplines. En fait, les sciences font encore partie de la philosophie: elles s'intègrent dans un tout qui est la philosophie. Aussi ne faut-il point s'étonner que les élèves de la classe de physique défendent des thèses de mathématiques devant le professeur de philosophie. ${ }^{19}$

A y regarder de près, la classe de logique est bien, pour reprendre une formule de Philippe Ariès ${ }^{20}$, une cellule de la

15 On se rappellera le mot de d'Alembert: "Locke créa la métaphysique à peu près comme Newton avait créé la physique."

16 Sur le cartésianisme au XVIIIe siècle, voir l'article de René Poirier dans le Dictionnaire des lettres françaises: le dix-huitième siècle (Paris, 1960), A-K: 268-273.

17 Plan d'éducation du Séminaire de Québec, octobre 1790, document CXV, in RUL (novembre 1958): 270.

18 Ibid: 272.

19 Voir l'intitulé des thèses de mathématiques et de physique soutenues en $1775,1790,1792,1796$ : ASQ Thèses 1775-1815.

20 Philippe Ariès, L'enfant et la famille sous l'Ancien régime (Paris, $1960)$, 189. La deuxième partie de cet ouvrage contient des vues très pertinentes sur l'histoire de l'enseignement: La vie scolastique, 143-376. 
structure scolaire: elle jouit d'une unité physique, d'un local spécialisé, elle constitue une étape dans l'acquisition progressive des connaissances, elle relève d'un professeur déterminé, elle est fréquentée par des élèves d'âges et de conditions variables: toutes choses dont la connaissance nous aiderait à comprendre ce qu'était hic et nunc l'enseignement de la philosophie.

Qu'il suffise de dire ici que la classe de logique a été "régentée" pour l'ordinaire par de jeunes prêtres de la maison et, parfois, par des séminaristes sur le point de recevoir les ordres. L'écart dans l'âge des écoliers semble avoir été assez considérable, si l'on en juge par un dénombrement fait en 1790. Cette année-là, la classe de logique comprend des étudiants de 24, 25 et 26 ans, à côté d'écoliers de 17, 18 et 19 ans. ${ }^{21}$ Rappelons aussi que les deux classes de philosophie contiennent un certain nombre d'ecclésiastiques: ce régime existe depuis déjà quelque temps lorsque Mgr Hubert écrit en 1793:

Le Séminaire de Québec se trouve tout à fait dépourvu d'ecclésiastiques; on a jugé qu'il serait convenable après les vacances de donner la soutane à ceux des philosophes qui se destinent à l'état ecclésiastique, lesquels cornmenceront leur grand séminaire tout en continuant leur physique. ${ }^{22}$

Les cahiers manuscrits laissés par les écoliers et les "régents de logique", ${ }^{23}$ de même que les thèses que les premiers soutinrent, nous renseignent encore sur l'enseignement de la philosophie.

Force nous est de reconnaître que jusqu'en 1790, et même audelà, on semble être allé au plus pressé. Les compendium abondent; la plupart s'arrêtent à la logique ou glissent rapidement sur la métaphysique et la morale.

21 ASQ, Fonds Viger-Verreau, Carton $16 \mathrm{~N}^{\circ} 10$. L'écart semble s'être réduit par la suite; voir l'appendice $\mathrm{A}$.

$22 \mathrm{Mgr}$ Hubert à M. Brassier, 5 août 1793: lettre copiée dans ASQ, Classes du Séminaire de Québec 1760-1795.

23 C'est ainsi qu'on désigne le professeur de la classe de logique: Plan d'éducation du Séminaire de Québec, octobre 1790, document CXV in RUL (novembre 1958) : 273. 
Les professeurs cultivent la brièveté, se font fort d'écarter les subtilités. L'un d'entre eux, l'abbé Robert, ouvre son cours en 1790 par une déclaration savoureuse:

Hoc vestri causa compendium redegi, carissimi philosophiæ alumni, non desunt quidem qui prolixiores lectiones exhibuerint, sed sæpius, nimia prolixitas aut fastidium parit, aut vires adolescentis superat. Brevitati consulens, nihil claritati auferam intricatas quæstiones omisi, ex quibus nullam aut parvam duntaxat utilitatem prævidi percepturas. ${ }^{24}$

On ne trouve point dans les introductions, qui sont généralement courtes, des considérations sur la philosophie d'Adam ou sur l'existence de la philosophie. D'aucuns s'impatientent même de ces discussions sur la vraie définition de la philosophie:

Maximam temporis partam quidam insumunt philosophi ut definitionem suam propugnant, et adversariorum reffellant [repellant] objectiones, sed hoc non maximi videtur momenti. ${ }^{25}$

Faut-il mettre sur le compte de cette volonté de chasser les superfluités la faible dimension des cahiers manuscrits ? Ou estce, comme le voulait Mgr d'Esgly, le résultat d'une dégénérescence des études?

Les sciences tombent ici à vue d'œil, écrit-il en 1785.

Les humanités, la rhétorique ne sont plus rien; la

philosophie dégénère beaucoup; la théologie même a beaucoup besoin qu'on l'aide. ${ }^{26}$

Chose certaine, vers cette époque, en France, les auteurs de traités et de manuels de philosophie bannissent de plus en plus arguties et ergoteries. Un historien de l'enseignement l'a fort bien noté:

En même temps qu'on écarte ou qu'on tempère peu

à peu dans la rédaction des traités, l'aridité de la sco-

lastique, on supprime un grand nombre de discus-

${ }^{24}$ ASQ M 185, page de couverture.

25 ASQ M 151 (1792), 17.

26 Cité par Lionel Groulx, L'enseignement français au Canada (Montréal, 1931), I : 49. 
sions subtiles que cette méthode avait trop longtemps soulevées sans grand profit pour l'esprit humain. ${ }^{27}$

Quoi qu'il en soit, les cahiers manuscrits dépassent rarement les cent pages. Que faisaient les écoliers ? Nous avons vu que dans la classe de logique, ils n'étudient que la philosophie proprement dite. Les compendium posent donc un problème délicat que nous ne pouvons éluder: c'est celui de l'emploi du temps.

Nous croyons que certaines années il n'y avait pas cours de philosophie tous les jours et que la durée des cours eux-mêmes variait. C'est du moins ce que nous laisse entendre un professeur qui a daté en 1782 les cours de philosophie qu'il a faits d'octobre à décembre. Les dates, en effet, ne sont pas successives et la longueur des notes varie beaucoup d'une datation à l'autre. ${ }^{28}$

Non contents de suivre les cours et de rédiger leurs cahiers, les écoliers soutiennent des thèses de philosophie. C'est le seul exercice que semble comporter l'étude de la philosophie. Les archives du Séminaire possèdent les textes manuscrits de thèses soutenues en 1772, 1774, 1777 (ou 1778), 1783 et 1797.

Trait bien scolastique, les écoliers se présentent deux à la fois, l'un devant attaquer, l'autre défendre la thèse. L'avis de convocation à la soutenance de thèses de 1772 est rédigé en un style singulièrement précis et sublime:

Has theses deo duce, et auspice dei para tueri conabuntur franciscus Perrault Josephus Verreau die $26^{\mathbf{e}}$ januarii anno 1772 hora post meridiem quinta usque ad sextam in Seminario quebecensi. ${ }^{29}$

Nous ignorons si, à l'exemple des soutenances de thèses de mathématiques et de physique, une certaine pompe entoure celles de philosophie. Il n'est pas interdit de le penser. En tout cas, une note sibylline laissée par l'abbé Charles Chauveau nous apprend qu'en 1783 ses élèves furent "argumentés par Monsei-

27 Sicard, op. cit., 267.

28 ASQ M 154.

29 ASQ Sem 13, $\mathrm{N}^{\circ} 14$. 
gneur". ${ }^{30}$ Cette année-là, les logiciens durent défiler devant Mgr Briand et répondre de leurs connaissances.

Dans la préparation de leurs cours, les professeurs s'inspirent surtout de deux manuels français.

Le premier, les Institutiones philosophicæ ad usum Seminarii Tullensis, ouvrage en cinq volumes, publié d'abord en 1763, puis réédité jusqu'à la Révolution, fut envoyé en 1766 au Séminaire de Québec par le Séminaire de Paris. ${ }^{31}$ Rédigé à l'origine pour les étudiants du diocèse de Toul, ce manuel se répandit dans

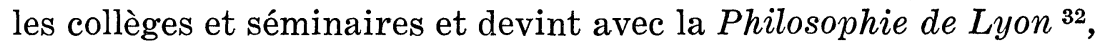
publiée en 1782, l'un des plus utilisés durant les années qui précédèrent la Révolution. De solides qualités recommandaient la Philosophie de Toul. Rigoureusement impartiale, elle offrait "non le résumé, mais l'exposé de toutes les discussions engagées depuis la réforme philosophique du XVIIe siècle". ${ }^{33}$ Ouvrage encyclopédique où, comme on le verra, les professeurs ne manqueront pas de puiser.

Un second manuel en deux volumes, d'esprit et de facture moins scolastiques, l'Abrégé latin de philosophie ${ }^{34}$, publié en

30 ASQ M 154, 38. Louis Bourdages et Pierre Bédard soutiennent des thèses en 1783, Ibid., 39. Bédard cultivera toute sa vie la philosophie et les mathématiques: les archives du Séminaire possèdent de lui un gros cahier de notes colligées après la fin de ses études. Voir l'article de Mgr Arthur Maheux: Pierre Stanislas Bédard, 1763-1829: philosophe et savant, in MSRC (troisième série) L (1956) : 85-93.

31 ASQ, Sém. 8, $\mathrm{N}^{\circ} 55$ a. Le texte parle de la "Philosophie de Camier": il s'agit du nom de l'un des deux auteurs de l'ouvrage. Plusieurs exemplaires de ce manuel viendront par la suite. Le Journal de dépense du Séminaire (1781-1809) signale pour juin 1784: "Philosophie de Camier par le régent de pphie." La bibliothèque de l'Université a conservé 4 éditions de la Philosophie de Toul; celle de 1763 provient de la bibliothèque de Mgr Briand.

32 Institutiones philosophicaæ auctoritate archiepiscopi Lugdunensis editæe, 1782, 5 vol. On ne semble pas avoir connu ce manuel à Québec.

33 A. Mignon, La philosophie cartésienne dans le clergé de France aux débuts du XIXe siècle, in Revue du clergé français, XX (1899), 138. L'auteur ajoute: “Thèses cartésiennes, opinions de Malebranche, controverses d'Arnauld avec Descartes, tout est placé dans une mesure qui permettait d'y trouver des renseignements nombreux sinon profonds."

34 Abrégé latin de philosophie avec une introduction et des notes françaises (Paris, 1784), 2 vol. L'ouvrage est souvent appelé Abrégé latin de Hauchecorne, du nom de son auteur. 
1784, fut aussi mis à contribution durant la dernière décennie du siècle. Bien que respectueux à l'endroit de Descartes ${ }^{35}$, l'auteur abandonne le système des idées innées pour l'empirisme de Locke. Il ne cache pas non plus son mépris de la philosophie scolastique:

Quelques commentaires d'Aristote, faits par des ignorants, ressuscitent le Péripatétisme; une dialectique subtile, un raffinement de chicanes, un cercle de disputes puériles, du bruit, des cris, un langage barbare; voilà ce que l'on voulut bien nommer la Philosophie Scholastique. ${ }^{36}$

Il n'est pas du tout impossible que ce manuel ait été introduit par l'un des prêtres français qui, chassés par la Révolution, enseignèrent la philosophie au Séminaire: les abbés Castanet, Raimbault et Desjardins. Les deux premiers utilisent copieusement l'Abrégé latin de Hauchecorne, comme on en peut juger par les cahiers manuscrits de leurs cours qui sont conservés aux Archives du Séminaire.

En plus de ces deux manuels, les professeurs peuvent consulter les nombreux ouvrages de philosophie dont est pourvue la bibliothèque du Séminaire, ainsi que l'atteste le catalogue préparé par l'abbé Dudevant en $1782 .{ }^{37}$ Une foule de manuels du XVIIe siècle et de la première moitié du XVIIIe siècle y figurent, ce qui montre que le Séminaire s'intéressa à la philosophie bien avant $1765 .{ }^{38}$ Les œuvres d'Aristote, de Bayle, de Locke, de Male-

35 "Descartes: voilà le génie créateur qui seul va réformer la philosophie." Ibid., I : XXXV.

36 Ibid., I: XXXI.

$37 \mathrm{ASQ}$ Catalogue des livres de la bibliothèque du Séminaire des Missions étrangères de Québec fait dans le mois de May 1782. Sur l'auteur et le catalogue, voir l'article de M. Antonio Drolet: La bibliothèque du Séminaire de Québec et son catalogue de 1\%82, in le Canada français, XXVIII (1940) : 261-266.

38 Rappelons que la bibliothèque des Jésuites ne sera remise au Séminaire qu'en 1797. Voir l'article de M. Antonio Drolet, La bibliothèque du Collège des Jésuites, in RHAF, XIV (1961): 487-545. Il est fort probable toutefois qu'un certain nombre de livres - surtout des manuels ayant appartenu aux Jésuites se soient glissés au Séminaire avant 1797. 
branche, de Rousseau et de Voltaire apparaissent aussi au catalogue. La Logique de Port-Royal s'y trouve, bien entendu. ${ }^{39}$

Serrons de plus près encore notre sujet. Le latin est la langue de l'enseignement. Cahiers et thèses sont tout entier rédigés en latin et suivant les canons de la méthode scolastique. Une démonstration ne se fait point sans appareil syllogistique et une minutieuse mise en place des arguments: probo - objicies respondeo - negoanteriorem (minorem aut majorem) - instas - respondeo - etc. La vigueur de la scolastique est visible aussi dans la nette insistance accordée aux formes de raisonnement. L'étude de l'ars syllogisticae et methodi occupe une place de choix dans l'enseignement; les thèses qui sont conservées aux Archives du Séminaire portent toutes sur la logique.

L'appellation de la classe de philosophie est elle-même riche de signification. Tout comme dans les collèges et séminaires français de l'époque, elle est dite classe de logique, sans doute par fidélité au vocabulaire introduit à la Renaissance par les Jésuites français, bien que depuis le milieu du XVIIIe siècle l'annexion de la métaphysique et de la morale ait rendu l'expression impropre.

En revanche, nous notons que sur le vieux fonds scolastique sont venus se greffer plusieurs thèmes cartésiens. Le cartésianisme et le vocabulaire cartésien ont pénétré l'enseignement. Certaines questions de logique que la scolastique avait de tout temps jugé fort importantes ne sont plus étudiées: les cahiers manuscrits ne parlent ni des universaux ni des prédicaments ou même des prédicables. ${ }^{40} \mathrm{Du}$ reste, la définition de la philosophie attribuée aux péripatéticiens est allégrement réfutée; la plupart des cahiers et des thèses retiennent la définition toute cartésienne que propose la Philosophie de Toul: "cognitio ex ratione discursiva." ${ }^{41}$

39 On se fera une idée plus précise des ouvrages de philosophie inscrits au catalogue de l'abbé Dudevant en consultant l'appendice 8.

40 Nous avons rencontré une exception: l'abbé Robert définit les cinq universaux, ASQ M151, 49. I: $18 \mathrm{~s}$.

41 Institutiones philosophicæ ad usum Seminarii Tullensis (éd. 1769), 
Un grand nombre de problèmes reçoivent des solutions cartésiennes, depuis la nature de la philosophie jusqu'à l'origine des idées. Les cahiers et les thèses répètent à l'unisson que l'évidence (à laquelle ils adjoignent parfois le sens intime) est le fondement (motivum) de la philosophie. Évidence qu'on définit à la suite de Descartes:

Clara et distincta perceptio habitudinis inter ideas seu clara visio rei in ipsa considerata. ${ }^{42}$

La logique elle-même - hormis l'inaltérable section du syllogisme - est envahie par le cartésianisme. Elle comprend quatre parties: la perception, le jugement, le discours et la méthode, qui correspondent aux quatre opérations de l'esprit:

Mens debet $1^{\circ}$ ideas comparare et ordinare. $2^{\circ}$ judicare. $3^{\circ}$ deducere conclusiones ex principiis cognitis. $4^{\circ}$ tandem cogitationes suas ordinare. ${ }^{43}$

Comme le veut la théorie cartésienne de l'intuition, les cahiers soutiennent que toute idée est nécessairement vraie, claire et distincte; le jugement est tenu pour un acte de la volonté, bien que par un curieux retour il devienne parfois le fait de l'intelligence ${ }^{44}$; le raisonnement et la méthode assurent, eux, le passage d'une vérité à une autre au moyen de la déduction, C'est-à-dire, de l' "illatio unius de alio per aliud" ${ }^{45}$.

Quatre règles, tirées du Discours de la méthode, président à la recherche de la vérité:

$1^{\circ}$ Nulli rei assensus præbeatur, nisi perspectæ.

$2^{\circ}$ Ne plura simul menti afferantur, sed implicita nimis distribuantur per partes, ne confusio fiat.

$3^{\circ}$ Maxime ponderantur judiciorum motiva, præcipue, quando stabiliuntur propositiones, tanquam principia, et caveatur sedulo ut conclusiones ex ipsis necessario sequantur.

$4^{\circ}$ Tandem sive disputemus, sive repellamus summo prosequamur odio quidquid arrogantiam aut adversarii contemptum edolet. ${ }^{46}$

${ }^{42}$ A SQ M 185 (1790), 10.

43 ASQ M 151 (1792), 47.

44 ASQ Sém 13, $\mathrm{N}^{\circ} 15,16$ : Thèses $(1774,1777$ ou 1778).

45 ASQ Sém $13 \mathrm{~N}^{\circ}$ 15: Thèse (1774).

46 ASQ M 151 (1792) $123 \mathrm{~s}$. 
Les cahiers et les thèses reviennent sans cesse sur l'origine et la nature des idées, deux grandes questions qui troublent les esprits et dont l'étude (chose très révélatrice: nous sommes loin ici de l'ancienne scolastique) relève de la logique. L'innéisme cartésien et l'empirisme de Locke sont tour à tour défendus, puis combattus, mais le plus souvent avec circonspection:

Non omnis (idea) oritur a sensibus, nulla a reflexione in sensationes, quinimo non datur idea sensationum. An aliqua sit idea innata, nec ne, incertum. Non datur res impossibilis. ${ }^{47}$

Incertum est utrum sint annon idea innata quædam, licet probabilius sit non dari quam dari ideas innatas. ${ }^{48}$

Durant la dernière décennie du XVIIIe siècle, les professeurs rejettent carrément l'innéisme, sans pour autant donner tout à fait dans l'empirisme de Locke. Leur perplexité apparaît en plus d'un passage:

Idea innatæ cartesii nullo probantur argumento et nunc ab omnibus derelictæ jacent; opinio Lockii suos habet difficultates et ab omnibus non propugnatur; ergo fatendum est idearum originem non multo clarius nobis innotescere quam ipsarum naturam..$^{49}$

Toujours soucieux de ne point s'embarrasser avec des riens, l'abbé Robert se défend ainsi d'avoir consacré de longs développements à la question de l'origine des idées :

Multi philosophi acriter inter se disputant, utrum detur idea nihili, necne, sed parvi momenti talis quæstio videtur. ${ }^{50}$

Une thèse soutenue en 1797 résume assez bien l'impatience et le scepticisme des esprits:

47 ASQ Sem $13 \mathrm{~N}^{\circ} 15$ : Thèse (1774). Deux ans plus tôt, une thèse défendait l'empirisme... "Nulla datur idea nihili, nulla rei impossibilis, nulla innata; partim a sensibus, partim a reflexione comparantur ideae." ASQ Sém $13 \mathrm{~N}^{\circ}$ 14: Thèse (1772).

48 ASQ Sem $13 \mathrm{~N}^{\circ} 16$ : Thèse (1777 ou 1778).

49 ASQ M 172 (1796), 7 s. Ce passage est tiré en entier de l'Abrégé latin de philosophie, I: $4 \mathrm{~s}$.

50 ASQ M 151 (1792), 51. 
Logica humana mentis operationes dirigit; circa naturam et originem idearum, frustra systemato systematibus opposuere philosophi ad huc sub judice est, nullam in re tam obscura opinionem propugnare multo tutius duximus quam multiplices hujus dedali meatus penatrare ex quo ut extricare nos possemus nulla paret quæ præstet arcana filum. ${ }^{51}$

Le problème de l'origine des idées est peut-être le seul où l'enseignement se fasse hésitant. Généralement, la philosophie est bien capable de connaître la vérité. On lui assigne communément le sujet suivant: "mens humana veritatis opprime tenax". ${ }^{52}$ La certitude n'est pas un idéal chimérique, puisque l'évidence, le sens intime, la mémoire, le témoignage des hommes et le rapport des sens constituent autant de gages de la vérité. Les professeurs se gardent toutefois de rejeter le doute méthodique pratiqué par les cartésiens; seul le doute réel (dubium effectivum aut serium), dont les funestes effets n'apparaissent que trop, est pourfendu:

... dubium scepticorum ... non est admittendum, ut pote impossibile veritatis inquisitioni, nocivum morum integritati, societatis securitati, religionis incolumitati perniciosum, denique homine sano indignum. ${ }^{53}$

Le pyrrhonisme historique n'échappe pas non plus à de vives attaques. Le problème devait présenter un certain intérêt, car les thèses soutenues en 1774,1777 , et 1783 contiennent des réfutations du pyrrhonisme. Les cahiers manuscrits s'y attardent aussi, complétant pour notre profit les démonstrations par de multiples exemples. L'un de ceux que présente l'abbé Robert en 1794 est emprunté à la plus récente actualité: "l'obstruncatio Ludovici decimi sexti", événement à propos duquel il ajoute aussitôt: "circa factum ajusmodi testes decipi non potuerunt". ${ }^{54}$

\footnotetext{
${ }^{51} \mathrm{~A} S Q$ Sem $13 \mathrm{~N}^{\circ}$ 17: Thèsse (1797).

$52 \mathrm{ASQ}$ Sem $13 \mathrm{~N}^{\circ} 14$ : Thèse $(1772)$.

53 ASQ M 154 (1783), 30: Thèse (1783).

54 ASQ M 175 (1790-1794), 61. Il n'est peut-être pas hors de propos de rappeler les réflexions de Colbert de Maulevrier de passage au Canada en 1798. L'ignorance du bon peuple "est telle, dit-il, que peu - au moment où j'écris - veulent croire à la mort du Roi de France. Il est caché, disent-ils et il reparaîtra, il a le pouvoir de se rendre invisible..." Voyages dans l'intérieur des Etats-Unis et au Canada (Baltimore, 1935), 66.
} 
Mais, si puissante soit-elle, l'autorité de l'évidence, du sens intime, de la mémoire, du témoignage des hommes et du rapport des sens, cesse devant le fait religieux qui tire sa certitude de la révélation divine:

His omnibus præmitte Divinam auctoritatem cui omnimode debetur assensus in lis quæ sunt supra Rationem. ${ }^{55}$

Prenons garde, toutefois; la révélation divine n'est pas plus certaine que l'évidence, la certitude théologique ne dépasse pas la certitude philosophique. De nettes formules expriment cette confiance en la raison:

Auctoritas divina ... licet non certior sit evidentia, fidem tamen meritur in lis quæ sunt supra rationem posita ... ${ }^{56}$

Revelatio ... divina non est certior evidentia, ideoque certitudo conclusionum theologicarum non est major certitudine conclusionum philosophicarum..$^{57}$

C'est surtout pour l'étude de la métaphysique que l'enseignement suit de près la Philosophie de Toul et l'Abrégé latin de Hauchecorne. La filiation devient évidente durant la dernière décennie du XVIIIe siècle. Le compendium de métaphysique générale préparé par l'abbé Robert en 1792 examine trois questions: de Essentia spirituum, de Existentia spirituum et de Causis spirituum, dans l'exposé desquelles il résume la Philosophie de Toul.58 L'abbé Castanet en 1795 suit fidèlement l'Abrégé latin de Hauchecorne, dont il extrait les questions suivantes: les trois preuves de l'existence de Dieu ${ }^{59}$, une discussion de l'athéisme, de longues réfutations des systèmes de Straton, d'Épicure, de Spinoza et des immatérialistes, et enfin une étude des attributs de Dieu. ${ }^{60}$

55 A.SQ Sém $13 \mathrm{~N}^{\circ} 15$ : Thèse (1774).

56 ASQ M 154 (1783), 32, Thèse (1783).

57 ASQ Sém $13 \mathrm{~N}^{\circ} 16$ : Thèse (1777 ou 1778).

58 ASQ M 151 (1792), 125-146 et Institutiones philosophicæ ad usum Seminarii Tullensis (éd. 1769), I: 348 ss.

21.

59 Dont la preuve cartésienne de l'être nécessaire, ASQ M 155 (1795),

60 Ibid., 1-86 et Abrégé latin de philosophie, I: 109 ss. 
Il arrive parfois, pendant cette décennie, que les cahiers manuscrits reproduisent d'adroites citations françaises. Relevons celle-ci de Jean-Jacques Rousseau, introduite par l'abbé Castanet dans sa démonstration de l'existence d'une vie future:

Plus je rentre en moi, plus je me consulte, et plus je lis ces mots dans mon âme, sois juste et tu seras heureux. Il n'en est pourtant rien, en considérant l'état présent des choses; le méchant prospère et le juste reste opprimé. Si l'âme est immatérielle, elle peut survivre au corps; et si elle lui survit, la providence est justifiée. Quand j'en aurais d'autres preuves de l'immatérialité de l'âme que le triomphe du méchant et l'oppression du juste, cela seul n'empêcherait d'en douter. Une si choquante dissonance dans l'harmonie universelle, me ferait chercher à la résoudre. Je me dirais, tout ne finit pas pour moi avec la vie; tout rentre en ordre avec la mort. ${ }^{61}$

Cette citation ne doit pas étonner. Dès avant la Révolution, l'influence de Rousseau fut mise au service de l'apologétique religieuse. Jean-Jacques n'est-il pas l'ennemi des Encyclopédistes, l'avocat d'une sorte de sentiment religieux ? Son œuvre devint "le bréviaire des honnêtes gens"; la société française du début du XIXe siècle vénéra Rousseau non moins que Chateaubriand et le Génie du christianisme.

De même qu'on ne voit en Rousseau que l'apologiste de la religion, de même n'enseigne-t-on qu'une partie de la philosophie de Descartes ou de Locke. Les cahiers manuscrits et les thèses, peut-être par souci d'éviter une question difficile, ne parlent point du cogito. On ne suit pas en Locke le penseur politique ou le théoricien du droit naturel, mais seulement le philosophe qui a résolu la délicate question de l'origine des idées; pour s'en convaincre, il suffit d'examiner ce que renferment les cahiers manuscrits sur le pouvoir et sur la société civile. En l'absence de thèses d'écoliers sur le sujet, nous nous sommes reporté à l'exposé

${ }^{61}$ ASQ M 155 (1795), $100 \mathrm{~s}$. La citation est tirée de la Philosophie de Toul (éd. 1769), II: 388 ss. Nous verrons plus loin que les cahiers manuscrits contiennent un grand nombre de citations françaises à partir de 1818; on invoquera alors fréquemment le témoignage de Rousseau. 
le plus complet, celui de l'abbé Robert. Nous y découvrons des idées très voisines de celles qu'a illustrées Bossuet. ${ }^{62}$

Divin par son origine (non est potesta nisi a Deo... Suprema civilis potestas a solo Deo, non autem a populo ducit originem) et donc sacré (qui resistit potestati Dei ordinationi resistit), le pouvoir confère un sacerdoce:

... quicumque auctoritatem politicam exercent Dei imagines sunt et ministri, ea in auctoritatis parte quam exercent. ${ }^{63}$

La rébellion ne se justifie en aucun cas, le prince fût-il cruel ou hostile à la vraie religion:

Nunquam licitum est subditis rebellare in politicam auctoritatum quamvis igitur princeps crudeliter imperaret, quamvis esset veræ religionis hostis infensissimus. ${ }^{64}$

De la souveraineté du peuple, il ne peut résulter que des désordres. Il répugne au bien de la société que le peuple soit juge des lois établies par le pouvoir civil et qu'il puisse les révoquer à sa guise. Du reste, les É critures le confirment:

Huic vestræ rationes doctrinæ concordat scriptura sacra: per me reges regnant et legum conditores justa decernunt. prov: (8. V. 15) ${ }^{65}$.

Après avoir distingué trois formes de gouvernement, la monarchie, l'aristocratie et la démocratie, et noté que le régime britannique est un alliage des trois, l'abbé Robert pose la question classique: laquelle de ces trois formes est la meilleure ? Il répond d'abord que nous devons défendre le régime sous lequel nous vivons, quel qu'il soit. Mais la plus estimable des formes de gouvernement est celle qui fait le plus fleurir la justice, l'amour de la patrie et la religion dans le cœur des citoyens. La monarchie, régime très ancien et paternel et donc plus conforme à la nature,

62 Jean-Jacques Chevalier, Les grandes æuvres politiques, de Machiavel à nos jours (Paris, 1957): 70-84.

63 ASQ M 175 (1790-1794), 369.

64 Loc. cit.

65 Ibid., 368. Souligné dans le texte. 
y est parvenue à un degré éminent. L'Histoire nous fait préférer la monarchie à la démocratie, régime dont l'institution est récente. ${ }^{66}$

Université Laval, Québec.

MARC LEBEL, B.A., B.PH.

N.B. On voudra bien se reporter à notre rubrique "Bibliographie" pour des notes complémentaires à cette étude. N.D.L.R.

${ }^{66}$ Loc. cit.

Articles à paraître dans notre prochaine livraison:

Dr Eduardo Brazao, ambassadeur du Portugal, Collaboration luso-danoise dans la découverte de l'Amérique du Nord.

Yves Poutet: Jacques II, Mac-Mahon et Kennedy.

J. LALONDE-RÉmillaRd: Angélique des Méloizes.

Bibliographie de: Ludger Duvernay et les débuts de la presse périodique aux Trois-Rivières.

ROLAND LAMONTAGNE: Le ministère de la marine dans ses rapports avec Mgr de Pontbriand. 